\title{
Relationship between oral candidiasis and users of dental prostheses
}

\author{
Ana de Lourdes Sá de Lira ${ }^{1}$, Aline Cardoso Torres ${ }^{1}$
}

Aim: To investigate the relationship between oral candidiasis and users of dental prostheses. Methods: A cross-sectional study was carried out which the inclusion of adults and elderly people of both genders using dental prostheses. Lectures were given with guidelines on oral hygiene techniques and dental prostheses, preventive measures of candidiasis, highlighting their influence on the oral and general health of the individual for the 240 users of dental prostheses. Of this number, 153 did not want to participate in the clinical examination. Signals and symptoms were analyzed in 87 patients, and lesions were present in only 21 , and a cytological complement of the lesions was performed. Data were analyzed using SPSS version 15.0 with Chi-square $\left(\chi^{2}\right)$ test at $p<0.05$. Results: The 21 patients presented oral candidiasis, with erythematous (prosthetic) stomatitis being the most prevalent. There was a correlation between the presence of Candida and the use of prostheses (p-value $0.00 *$ ). Candidiasis was associated with poorly adapted prostheses, poor oral hygiene, inadequate prostheses and prolonged use of them. Conclusion: There was a correlation between oral candidiasis and users of dental prostheses. The most prevalent type of candidiasis was erythematous stomatitis (prosthetic), and nystatin was used to treat all cases. The preventive activities carried out were extremely important, since patients were not aware of oral candidiasis and the factors that trigger it, such as poor oral hygiene and dental prostheses.

Keywords: Candidiasis, oral. Dental prosthesis. Candida albicans. 


\section{Introduction}

The higher prevalence of different Candida species in denture weares in contrast to no denture weares demonstrated that denture insertion induces plaque formation favoring the increased population of potentially pathogenic bacteria and Candida spp. The most of the denture plaque occurs on the denture-base of the upper denture, and hence the tissue contact surface of the upper denture was considered for isolation of Candida?.

The most prevalent fungal infection in the oral cavity of humans is oral candidiasis or candidosis. It mainly affects the jugal mucosa, palate and back of the tongue. The fungus responsible for this disease is of the genus Candida, and there are several types of the same species, for example: C. tropicalis, C. glabrata, C. krusei and C. albicans. However, the species $C$. albicans is the most present and pathogenic of all species of the genus Candida1-4.

Among the main etiological factors for the development of candidiasis infection are immunosuppressive diseases such as HIV and AIDS, cancer treatments, organ transplants, diabetes, use of corticosteroids and antibiotic therapy. It is an opportunistic disease and depending on the state of the host can manifest as bacterial sepsis and spread through several organs causing morbidity or even death of the individual 5 .

Oral candidiasis can be divided into pseudomembranous which is characterized by the appearance of removable white plaque, erythematous by the presence of erythema, Occurring more frequently on the palate and dorsum of the tongue, and may or may not have small petechiae or granules scattered throughout the affected region. This is the typical form found in elderly patients with total or partial dentures, known as stomatitis Prosthetic. Hyperplastic manifestation is less common with white lesions due to hyperkeratosis, and are unstable by scraping. The mucocutaneous is characterized by the presence of white plaques and reddish areas ${ }^{3,4}$.

The use of dental prosthesis associated with poor hygiene, both buccal and prosthesis, are also important local etiological factors ${ }^{4}$. People who use removable prostheses are more likely to have candidiasis compared to individuals who do not use them because of the ease of adhesion of microorganisms to acrylic prostheses, leading to the development of infection ${ }^{6}$.

Mechanical factors are also related to oral candidiasis, such as: loss of vertical dimension of occlusion with flaccidity of the upper lip over the lower one at the angle of the mouth, absence of teeth and use of maladaptive prostheses, the elderly population being more likely to get this infection. Generally they tend to develop successive infections in short intervals, therefore, it is necessary to take therapeutic measures to avoid recurrence of the disease $e^{7-9}$

The importance of prevention, early diagnosis and immediate treatment of the present oral lesions is emphasized with dissemination of knowledge of good oral hygiene habits, promoting educational actions, emphasizing the importance of hygiene of dental prostheses, through materials and appropriate techniques. The alkalinization of oral $\mathrm{pH}$ and use of antifungal drugs are effective behaviors adopted in the treatment and control of the condition, with nystatin being the most commonly used drug ${ }^{10}$. 
The aim of this research was to investigate the relationship between oral candidiasis and users of dental prostheses.

It is believed that oral candidiasis is directly related to maladaptive dentures or in poor conditions due to the long time of use, or precarious oral hygiene.

\section{Material and methods}

A cross-sectional study was carried out which the inclusion of adults and elderly people of both genders using dental prostheses. The sample totaled 240 people, after the sample calculation, who were attended monthly by the family health strategy doctor in the Basic Health Unit (BHU) of the district Planalto and Samaritana in the city of Parnaíba-PI. These patients had already had dental consultations. The descriptors used were: oral candidiasis, dental prosthesis and Candida albicans. For the review of literature and discussion, searches were indexed in the databases LILACS, MEDLINE, SCIELO, BIREME, PUBMED.

The researchers were provided with the letter of consent of the nurses heads of the Basic Health Units (BHU) in the city of Parnaíba, Piauí, after authorization to carry out the research development and approval of the Research Ethics Committee of the State University of Piauí, on the basis of opinion 1665.758.

Oral lectures with emphasis on preventive measures and early diagnosis (Figure 1) on oral candidiasis, its etiological factors and consequences were conducted biweekly during the year of the research, coinciding with the day of routine medical care with the Oral Health Strategy (BHU). Then, the Free and Informed Consent Form (FICF) was given to each patient, and the identification, health and oral hygiene and the prosthesis questionnaire were applied to be drawn the profile of each research member. The questionnaire was applied with fifteen objective questions contained demographic information such as age, sex, time of wearing, and other questions to know the attitude of denture hygiene habits, frequency of cleaning, and nocturnal denture wearing habits.
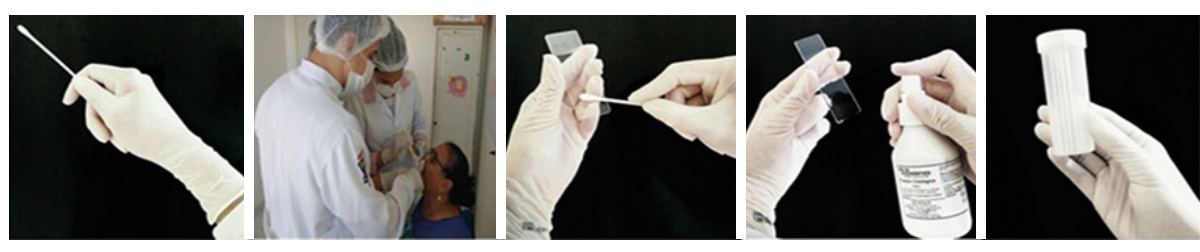

Figure 1. Scraping with swab(1), application of the material(2) and fixation with cytological fixative(3) and storage of the blade in blade holder(4).

Two researchers were trained at the Clinical School of Dentistry (CSD) of the State University of Piauí to identify possible lesions of oral candidiasis. Subsequently, calibration exercises were performed with 11 persons not participating in the sample plan, in a BHU of the municipality, according to the methodology described in another publication ${ }^{10}$. The pilot study involving 30 elderly people was carried out with the objective of testing the proposed methodology. As a result, its viability was observed, without adjustments. 
In order to assess intra- and inter-examiner diagnostic reproducibility, $10 \%$ of the total sample was double-examined by each of the examiners, with the Kappa coefficient obtained for intra and inter-examiner agreement 0.97 and 0.91 , respectively. Of the 240 patients who participated in the lectures, only 87 patients ( 55 elderly - $\geq 60$ years and 32 adults) users of dental prostheses were performed oral examinations in the $\mathrm{BHU}$. Among the reasons that justify the non-participation of the others stand out shame when removing the prosthesis, lack of interest and availability of time.

The objective of the examination was to investigate the possible presence of candidiasis, prosthetic conditions and the need for treatment, using the clinical examination method (signs and symptoms) using natural light and a led flashlight for better visualization, gloves for procedures and wooden spatula and complementary examination (exfoliative cytology) $)^{9,11 .}$

White lesions, when easily removed, are pathognomonic signs of pseudomembranous candidiasis. Cytological examination was performed in these 21 patients, regardless of the type of lesion present, although some authors 9,10 affirm that in the red lesions the yeast and filamentous forms of candidiasis are generally not present, and cytology is not necessary.

The sites where these lesions were detected were previously dried with sterile gauze and scraped with sterile Swab for collection (Dalian Goodwood Wooden Ltd, Youjiacun Xinzhaizi Town, Dalian, China).

After swab scraping, the smear was deposited on the blade (CRAL Disposable Labware Products Ltd, Cotia, São Paulo, Brazil), in a single movement from left to right so as not to damage the harvested material, which was then fixed with cytological fixative (Figure 1).

The slides were stored in the slide holder (Deskarplas disposable products, Mogi das Cruzes, São Paulo, Brazil) and sent to the laboratory to be stained using the Papanicolaou - Papanicolaou stain or Harris hematoxylin (Specialized Chemistry Erich Ltda, São Paulo, Brazil) and visualized in binocular biologic electronic microscope (M50A, Nikon Instruments Inc, Melville, New York, United States of America)' ${ }^{1}$. After the staining process, the clinical analyzes were performed for laboratory diagnosis, in the laboratory of the Federal University of Piauí (Campus Ministro Reis Veloso).

The diagnosis of oral candidiasis was based on clinical signs and symptoms, aided by microscopic examination of the smear. Patients who presented lesions, independently of the laboratory report, were treated with nystatin, under the supervision of dental surgeons of the UBH, for having exclusive effect on Candida and absence of systemic effects ${ }^{11-13}$.

Based on Ferreira et $\mathrm{al}^{12}$. protocol the patients were oriented to use disinfectant solutions to clean dental prosthesis and found that $\mathrm{NaOCl}$ at $0,5 \%$ was the most effective in the adhesion control of Candida yeasts and they also were oriented to remove the dental prosthesis during sleep, because of the relaxation and rest of the tissues, including the cleaning action executed by the saliva, tongue and cheeks are fundamental to maintain the oral mucosa healthy.

The measures adopted were as follows: Prescription Nystatin oral suspension (Micostatin ${ }^{\circledR}$ 100,000 IU / ml) -Blood for 1 minute and swallow $5 \mathrm{ml}$, 4 times daily for 
7 to 15 days until lesion remission; oral hygiene instruction; guidance on the chemical disinfection of prostheses with water solution and sodium hypochlorite $(0.25 \%)$, in the proportion of $1 \mathrm{ml}$ to $10 \mathrm{ml}$ of drinking water, for half an hour, twice a week, at least; suggestion for nocturnal resting of the prostheses and making new prostheses if necessary. In the removable partial dentures, the hygiene was performed with neutral soap, dental brush, and placed in a glass with water during sleep ${ }^{14}$.

A descriptive analysis of the data was performed using frequency and number tables using Microsoft Windows Excel 2013 software. The results were compared using the nonparametric chi-square $(X 2)$ test at a significance level of $5 \%(p<0.05)$ in the statistical package SPSS version 15.0 (Social Package Statistical Science).

\section{Results}

Regarding the sociodemographic situation of the research, the 240 members of the research participated in the lectures and answered the questionnaire. Their mean age was 59.20 years $(S D=13.02)$. Of these, $86.6 \%(n=208)$ were of the female gender and $13.33 \%(n=32)$ of the masculine gender who declared having a monthly income of $R \$ 1.093(S D=67.20)$.

Of the 87 people who were willing to perform the oral exam, 70 were female and 17 male. Of these, $21.25 \%(n=51)$ reported frequent use of antibiotics, but only $13.33 \%$ $(n=32)$ did so only by prescription. In addition, $10 \%(n=24)$ reported consuming alcoholic beverages, $7.08 \%(n=17)$ smoked and $6.25 \%(n=15)$ had any sexually transmitted diseases (STDs).

Regarding chronic diseases, it was observed that $44.58 \%(n=107)$ reported having hypertension, followed by allergic problems respectively $15.83 \%(n=38)$, diabetes and anemia $12.91 \%(n=31)$, respiratory problems $11.25 \%(n=27)$ and heart problems $7.91 \%(n=19)$.

About the oral hygiene conditions of the total sample, the majority reported adequate hygiene of $96.25 \%(n=231)$, but it was reported that they did not use dental floss and only removed their dentures to sanitize them once daily, being the majority with toothpaste and water $(n=165)$ and the others with neutral soap and water $(n=75)$. In addition, a large number of participants do not remove the prosthetic devices to sleep $(n=210)$, and those who do so place them in a glass of water.

Regarding the type of dental prosthesis used, it was observed that the prosthetic combinations were: removable partial prosthesis (RPP) in both arches in 75\% $(n=180)$, superior total prosthesis (TP) associated with the lower RPP in 17.08\% $(n=41)$ and TP in both arches in $7.92 \%(n=19)$. However, $17.5 \%(n=42)$ reported feeling some discomfort due to their poor adaptation. Cracks were observed in $8.33 \%(n=20)$ and spots in $4.58 \%(n=11)$ of the prostheses. When asked which professional had made the prosthesis, most did not know how to inform. However 30\% $(n=72)$ reported that it was the dental surgeon and $15 \%(n=36)$ the prosthetic.

In descending order, the oral clinical examinations made possible to find the following pathologies: caries, periodontal disease, tasty tongue, residual roots, tongue lost and ulcers. Of the 87 examined 21 (24.14\%) had lesions of candidiasis, 14 of them female, with ages between 47 and 65 years and 11 of the male, with age between 60 and 72 . 
Among the women 12 they used RPP superior and inferior. All males and two females used upper TP and lower RPP. There was a correlation between the presence of Candida and the use of prostheses $\left(p\right.$ - value $\left.0.00^{\star}\right)$. Prosthetic stomatitis was found only on female gender.

Oral lesions were found on the cheeks, tongue, palate and labial commissure. The distribution of the types of candidiasis found was demonstrated in Table 1 and Figure 2, after the diagnosis, evaluating the signs and symptoms of the disease, since the hyphae and spores were found in only 3 smears, suggestive of pseudomembranous candidiasis (Figure 3).

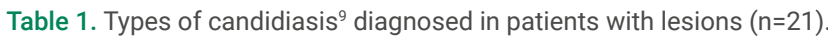

\begin{tabular}{lccccc}
\hline Types of candidiasis & Women & Men & Total & $\begin{array}{c}\text { \% of 87 } \\
\text { examined }\end{array}$ & p-value \\
\hline Pseudomenbranous & 2 & 1 & 3 & 3.45 & $0.02^{*}$ \\
\hline Hiperplastic & 1 & 1 & 2 & 2.3 & 0.05 \\
\hline Mucocutaneous & - & 1 & 1 & 1.15 & $0.01^{*}$ \\
\hline Acute atrophic erythematous & 1 & -- & 1 & 1.15 & $0.01^{*}$ \\
\hline Erythematous (Prosthetic stomatitis) & 10 & 1 & 11 & 12.64 & $0.00^{*}$ \\
\hline Chronic multifocal Erythematous & 3 & -- & 3 & 3.45 & $0.02^{*}$ \\
\hline
\end{tabular}

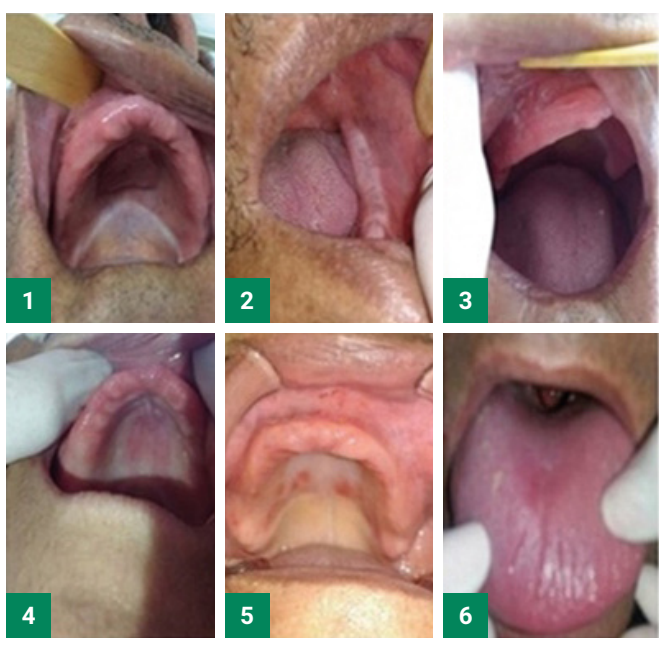

Figure 2. Horizontal sequence of images of the types of oral candidiasis found: Hiperplastic(1); Acute atrophic erythematous(2); Chronic multifocal Erythematous(3); Mucocutaneous(4); Erythematous (Prosthetic stomatitis)(5); Pseudomenbranous(6).
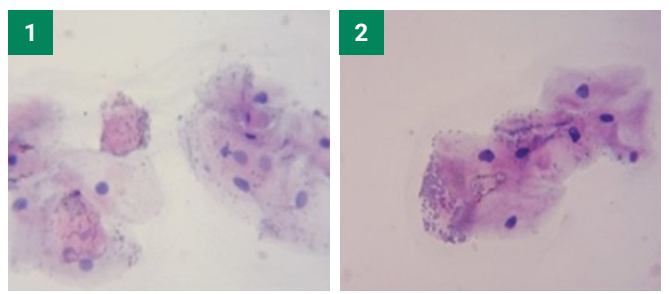

Figure 3. Blade smear with hyphae(1) and spores(2). 


\section{Discussion}

It is believed that oral candidiasis can affect quality of life and make feeding difficult due to the presence of bleeding, exudation, burning sensation, pain, halitosis, unpleasant taste and dry mouth, corroborating with other authors ${ }^{15-18}$.

According to the questionnaire, the exposure of the target public to the various predisposing factors of candidiasis, such as: antibiotic therapy, smoking, STDs, diabetes, anemia, respiratory problems, which depending on the individual's condition may lead to the development of the disease ${ }^{4}$.

Maladaptive prosthetics with cracks or spots are considered irritating factors for the buccal mucosa and may cause several lesions such as prosthetic stomatitis, inflammatory fibrous hyperplasia, inflammatory papillary hyperplasia, angular cheilitis and traumatic ulceration and cadidiasis ${ }^{14}$.

In the clinical examination, it was also observed that all the patients with lesions had caries, periodontal disease, tasty tongue and residual roots. According to Salerno et al. ${ }^{16}$, bacteria, such as Streptococcus spps, induce the formation of proteases that degrade the epithelium and cause inflammation, facilitating bacterial proliferation and colonization by Candida spp. Periodontal disease is also directly associated with candidiasis because it allows the colonization of fungi and fungi, increasing the chances of infection².

All 240 participants in the study used dental prostheses in the period between 11 and 20 years, extrapolating the average life span of 5 years $^{15}$. Some people said they felt discomfort from the use of the prosthesis, others reported maladaptation, besides showing cracks and blemishes. Such facts were observed by other authors ${ }^{7-9,14}$ who recommended a periodic visit to the dental surgeon to evaluate the adaptation, hygiene of the prostheses and the need to replace them.

The prerequisites for prosthetic assessment are retention, stability, reciprocity, fixation and aesthetics. If one of these requirements is not obeyed, the prosthesis should be replaced. Usually patients, in self-assessment, prioritize aesthetics, chewing and speech. When one of these factors is compromised, they are interested in looking for the dental surgeon ${ }^{16,17}$. It was observed in this research the lack of knowledge of the participants on the importance of the observance of prosthetic quality for oral health such as on others studies ${ }^{17-19}$.

Poor oral hygiene associated with the use of dental prosthesis predisposes to the development of buccal candidiasis ${ }^{6,7,13}$. Regarding the hygiene of the prostheses, most of them performed with a toothbrush and toothpaste, but it can cause roughness on the surface of the prosthesis, facilitating the creation of biofilms. Thus, the recommended method for sanitizing TP is the $0.25 \%$ sodium hypochlorite solution because it reduces biofilm with the remission of candidiasis ${ }^{18}$. Most reported not removing the prosthesis to sleep. Those who remove it put it in a glass with water, which is the most correct for conditioning, because when it is in a dry environment it suffers contraction, making it difficult to adapt it to the mouth ${ }^{19}$.

Prosthetic erythematous stomatitis was the most prevalent (12.64\%) characterized by extensive reddish areas on the palate, followed by chronic multifocal erythematous $(3.45 \%)$ with reddish atrophic spots and several subclinical types ${ }^{6}$. The lack of oral hygiene and prostheses promote biofilm formation and the colonization of Candida 
albicans ${ }^{9}$, which suggests being responsible for the high prevalence of prosthetic stomatitis. similar results were found by other authors ${ }^{7,19,23}$.

According to the literature, in the suspected hyperplastic candidiasis biopsy is recommended, but for the mucocutaneous and erythematous types, the indicated test is the culture medium, and the material harvested from the prosthesis. However, the most commonly used diagnostic method when any type of candidiasis is suspected is the clinical examination, the removal of predisposing factors, the use of antifungal agents until remission of the disease, and the need for complementary tests ${ }^{9}$.

Pseudomembranous candidiasis was found in $3.45 \%$ of the subjects, characterized by removable plaques with gauze or spatula, burning sensation and bad breath. Accompanying mainly immunosuppressed people. The clinical diagnosis is what differs this type from the others found. However, through the smear on the lamina, filamentous (hyphae) and spore forms were observed in three smears, typical of pseudomembranous candidiasis, corroborating with other authors ${ }^{6,11}$. This examination is recommended according to Schiboch et al..$^{10}$ (2005) when pseudomembranous candidiasis is suspected and it is possible to visualize under the microscope, after smearing and staining of the slides, fungi in yeast and filamentous forms with epithelial cells.

Hyperplastic candidiasis, found in $2.3 \%$ of the patients examined, is characterized by an asymptomatic non-removable thick white plaque, mainly found in the anterior region of the jugal mucosa and dorsum of the tongue, whose main etiological factor is smoking $^{6,20}$. Its diagnosis was obtained by clinical examination associated with administration of antifungal for 15 days for both patients and if it had not regressed, the biopsy would be performed. This is because this type of candidiasis may be associated with epithelial dysplasia called leukoplakia. In these cases, biopsy is recommended and should be considered a true dysplasia, with the possibility of becoming a malignant lesion ${ }^{12}$.

Mucocutaneous candidiasis was present in one of the participants with candidiasis, characterized by white plaques that stood out during scaling, exposing reddish areas. His diagnosis was confirmed by clinical examination and remission of the lesions after the use of antifungal for 20 days. The main predisposing factors are immune dysfunctions and the use of antibiotics. It can reach several organs and become systemic, leading to death. As noted in the questionnaire, there is a lack of knowledge about candidiasis. However, it is notorious that there is a need to pass on guidelines on preventive measures and their consequences ${ }^{21,22}$.

Chronic multifocal erythematous (2.45\%) and acute atrophic erythematosa $(1.15 \%)$ are subdivisions of erythematous candidiasis that were diagnosed in the study. The chronic multifocal type was presented by red macules on the palate and burning sensation. This type mainly affects the individuals with immunosuppression and who do antibiotic therapy, which was verified in the 3 treated patients. The acute atrophy presented as red atrophic mucosal areas due to atrophy of the papillae. The main etiologic factor of this type is immunosuppression ${ }^{23}$, which was reported by the patient with atrophic candidiasis.

Conventional treatment, which implies the application of topical antifungal drugs, provides an effective response, but there is a frequent occurrence of recurrence of the disease due to its multifactorial etiology, which is caused mainly by lack of oral hygiene, in addition to low immunity and person-to-person contamination. Another 
important factor in the recurrence of Candida albicans is deficiency in the hygiene of the prosthesis. Most microorganisms adhere to the acrylic surfaces, demonstrating a great mechanical resistance, especially in poorly adapted prostheses ${ }^{12,14,24,25}$.

Nystatin is one of the reference antifungal agents for the treatment of candidiasis, because it has no side effects or interactions with other drugs, with specific action against fungi of the genus Candida ${ }^{12}$. Therefore, it was the drug of choice for the treatment of patients, and it was obtained from UBS pharmacies as oral suspension (Micostatin ${ }^{\circledR}$ 100,000 IU / ml), 5ml, 4 times a day, and was chewed for 1 minute and then swallowed for 15 days, except those with hyperplasia, who used the drug for 20 days, as suggested by some authors ${ }^{14,16,21,22}$. Patients were supervised during treatment and up to 90 days after lesion remission. The users of badly adapted or compromised prostheses were referred to make new prostheses after treatment.

New research on the pathologies caused by the use of dental prosthesis as a factor predisposing to the alteration of oral microflora are important for diagnosis and reduction of complications.

It was concluded that there was a correlation between oral candidiasis and users of dental prostheses, the most prevalent type being erythematous (prosthetic stomatitis).

The preventive activities carried out were extremely important, since patients were not aware of oral candidiasis and the triggering factors, such as poor oral hygiene and dental prostheses. Treatment was performed with nystatin and all participants in the sample were guided on local and systemic predisposing factors (baseline disease), so that relapses were avoided. Individuals with lesions caused by removableprosthesis must be offered correct treatment which includes new prosthesis or adjustments of the old one. Periodical visits to the dentist are mandatory to prevent oral health problems. Research on the pathologies caused by the use of dental prosthesis as predisposing factor for oral microflora alteration are important for diagnosis and reduction of complications.

\section{References}

1. Andreola AD, Galafass EBE, Elsemann. [Comparative study between the production of extracellular phospholipases and proteinases of the genus Candida isolated from oral cavity infections]. Rev Odontol UNESP. 2016 Jul/Aug;45(4):219-26. Portuguese.

2. Lourenço AG, Ribeiro AERA, Nakao C, Motta ACF, Antonio LGL, Machado AA, et al. Oral Candida spp carriage and periodontal diseases in HIV infected patients in Ribeirão Preto, Brazil. Rev. Inst Med Trop. 2017 Mar;59(29):1-7. doi: 10.1111/odi.12669.

3. Ezenwa BN, Oladele RO, Akintan PE, Fajolu IB, Oshun PO, Oduyebo OO, et al. Invasive candidiasis in a neonatal intensive care unit in Lagos, Nigeria. Niger Postgrad Med J. 2017 Jul-Sep;24(3):150-4. doi: 10.4103/npmj.npmj_104_17.

4. Miyazima TY, Ishikawa KH, Mayer MPA, Saad SMI, Nakamae AEM. Cheese supplemented with probiotics reduced the Candida levels in denture wearers- RCT. Oral Dis. 2017 Oct;23(7):919-25. doi:10.1111/myc. 12680 .

5. Chanda W, Joseph TP, Wang W, Padhiar AA, Zhong M. The potential management of oral candidiasis using anti-biofilm therapies. Med Hypotheses. 2017 Sep;106:15-18. doi: 10.1016/j.mehy.2017.06.029 
6. Bianchi CM, Bianchi HA, Tadano T, Paula CR, Hoffmann-Santos HD, Leite Jr DP, Hahn RC. Factors related to oral candidiasis in elderly users and non-users of removable dental prostheses. Rev Inst Med Trop Sao Paulo. 2016 Mar;58(17):1-5. doi: 10.1590/S1678-9946201658017.

7. Przybyłowska D, Rubinsztajn R, Chazan R, Swoboda-Kopeć E, Kostrzewa-Janicka J, Mierzwińska-Nastalska E. The prevalence of oral inflammation among denture wearing patients with chronic obstructive pulmonary disease. Adv Exp Med Biol. 2015 Mar; 858:87-91. doi: 10.1007/5584_2015_128.

8. CARLI JP, Giaretta BM, Vieira RR, Linden MSS, Ghizoni JS, Pereira JR. [Oral lesions related to the use of removable dentures]. Rev Salusvita. 2013 Mar;32(1):103-15. Portuguese.

9. Lewis MAO, Williams DW. Diagnosis and management of oral candidosis. Br Dent J. 2017 Nov;223(9):675-81. doi: 10.1038/sj.bdj.2017.886.

10. Shiboski $\mathrm{CH}$, Chen H, Secours R, Lee A, Webster-Cyriaque J, Ghannoum M et al. High accuracy of common HIV-related oral disease diagnoses by non-oral health specialists in the AIDS clinical trial group. PLoS One. 2015 Jul 6;10(7):e0131001. doi: 10.1371/journal.pone.0131001.

11. Sahu A, Gera P, Pai V, Dubey A, Tyagi G, Waghmare M, et al. Raman exfoliative cytology for oral precancer diagnosis. J Biomed Opt. 2017 Nov;22(11):1-12. doi: 10.1117/1.JBO.22.11.115003.

12. Vasconcelos LC, Vasconcelos LCS, Ghersel ELA, Veloso DJ, Cunha PASM. Denture hygiene: importance in denture stomatitis control. RGO. 2013 Apr/Jun;61(2):255-61.

13. Sahu A, Sneha Tawde S, Pai V, Gera P, Chaturvedi P, Naird S and Krishna CM. Raman spectroscopy and cytopathology of oral exfoliated cells for oral cancer diagnosis. Anal Methods. 2015 Jul;(7):7548-59. doi: 10.1039/C5AY00954E.

14. Russo A, Carriero G, Farcomeni A, Ceccarelli G, Tritapepe L, Venditti M. Role of oral nystatin prophylaxis in cardiac surgery with prolongedextracorporeal circulation. Mycoses. 2017 Dec;60(12):826-9. doi: 10.1111/myc.12680.

15. Silva NP, Borges-Paluch LR, Cerqueira TPS, Vila Nova MX, Costa TBA, Jacobi CCB. Prevalence of Candida in oral cavity of patients wearing dental prosthesis. Mundo Saude. 2015;39(3):325-32.

16. Salerno C, Pascale M, Contaldo M, Esposito V, Busciolano M, Milillo L, et al. Candida-associated denture stomatitis. Med Oral Patol Oral Cir Bucal. 2011 Mar;16(2):139-43.

17. Colussi CF, Patel FS. [Use and Need for Dental Prosthesis in Brazil: Progress, Prospects and Challenges]. Saude Transf Social. 2016 Apr;7(1):41-8. Portuguese.

18. Costa RS, Filho HBLF, Chaves FN, Vasconcellos AA. [Lesions associated with the use of partial or complete removable dentures - features and treatment]. Prosth Labor Sci. 2015;5(17):62-8. Portuguese.

19. Celakil T, Baca E, Topcuoglu EN, Röhling BG, Evlioglu G, Özcan M. Prevalence of Candida albicans and Streptococcus aureus on maxillary obturators, maxillary defects and in saliva. A cross-sectional study. 2017 Jul/Sep;20(3):45-51. doi: 10.14295/bds.2017.v20i3.1366.

20. Lause M, Kamboj A, Fernandez Faith E. Dermatologic manifestations of endocrine disorders. Transl Pediatr. 2017 Oct;6(4):300-12. doi: 10.21037/tp.2017.09.08.

21. Veverka KK, Feldman SR. Chronic mucocutaneous candidiasis: what can we conclude about IL-17 antagonism? J Dermatolog Treat. 2017 Nov;21:1-6. doi: 10.1080/09546634.2017.1398396.

22. Li J, Vinh DC, Casanova JL, Puel A. Inborn errors of immunity underlying fungal diseases in otherwise healthy individuals. Curr Opin Microbiol. 2017 Nov;9(40):46-57. doi: 10.1016/j.mib.2017.10.016.

23. Paraguassú GM, Pimentel PA, Santos AR, Gurgel CAS, Sarmento VA. Prevalence of oral lesions associated with use of removable dental prostheses in a Stomatology service. Rev Cubana Estomatol. $2011 \mathrm{Jul} / \mathrm{Set} ; 48(3): 268-76$ 
24. Simões RJ, Fonseca P, Figueira MH. [Oral infections by Candida spp]. Odontol Clín-Cient. 2013 Jan/ Mar;12(1):19-22. Portuguese.

25. Prakash B, Shekar M, Maiti B, Karunasagar I, Padiyath S. Prevalence of Candida ssp. Among healthy denture and nondeture wearers with respect to hygiene and age. J Indian Prosthodont Soc. 2015 Jan-Mar;15(1):29-32. doi: 10.4103/0972-4052.155041. 